\title{
Microscale Inhomogeneity of Brain Tissue Distorts Electrical Signal Propagation
}

\author{
Matthew J. Nelson, ${ }^{1,2}$ Clémentine Bosch, ${ }^{3,4}$ Laurent Venance, ${ }^{3,4}$ and Pierre Pouget ${ }^{1}$ \\ ${ }^{1}$ Centre de Recherche de l'Institut du Cerveau et de la Moelle Epinière, Inserm UMRS 975, CNRS 7225, Université Pierre et Marie Curie, Hôpital de la \\ Salpêtrière, 75651 Paris, France, ${ }^{2}$ California Institute of Technology, Pasadena, California 91125, ${ }^{3}$ Team Dynamic and Pathophysiology of Neuronal \\ Networks, Center for Interdisciplinary Research in Biology, CNRS UMR 7241/Inserm U1050, College de France, 75005 Paris, France, and ${ }^{4}$ Université Pierre \\ et Marie Curie, ED 158, 75005 Paris, France
}

Interpretations of local field potentials (LFPs) are typically shaped on an assumption that the brain is a homogenous conductive milieu. However, microscale inhomogeneities including cell bodies, dendritic structures, axonal fiber bundles and blood vessels are unequivocally present and have different conductivities and permittivities than brain extracellular fluid. To determine the extent to which these obstructions affect electrical signal propagation on a microscale, we delivered electrical stimuli intracellularly to individual cells while simultaneously recording the extracellular potentials at different locations in a rat brain slice. As compared with relatively unobstructed paths, signals were attenuated across frequencies when fiber bundles were in between the stimulated cell and the extracellular electrode. Across group of cell bodies, signals were attenuated at low frequencies, but facilitated at high frequencies. These results show that LFPs do not reflect a democratic representation of neuronal contributions, as certain neurons may contribute to the LFP more than others based on the local extracellular environment surrounding them.

\section{Introduction}

The extracellular environment in the brain is typically considered to be and is modeled as a homogenous conductive milieu (Nunez and Srinivasan, 2006; see also Gold et al., 2006; Lindén et al., 2011). However, on a microscale, extracellular space is unequivocally not homogenous, which is a point that quickly becomes apparent upon viewing brain tissue under a microscope. Very little of the extracellular space is actual "space"; rather it is mostly comprised of neural structures such as glial cells, neuronal cell bodies, axonal fiber bundles, dendritic structures, blood vessels, and other inhomogeneities. Indeed extracellular fluid is thought to comprise only $12-25 \%$ of the brain's volume (Peters et al., 1991; Braitenberg and Schuez, 1998).

This homogeneity assumption shapes the interpretation of extracellularly recorded data, particularly local field potentials (LFPs). Under this assumption, electrical current and potentials are viewed as propagating freely in all directions from all neural sources, as would happen if the neural sources were suspended in a saline solution at the same positions. The LFP at a given point in space then

Received July 22, 2012; revised Nov. 1, 2012; accepted Dec. 9, 2012.

Author contributions: M.N., L.V., and P.P. designed research; M.N. and C.B. performed research; M.N. analyzed data; M.N., C.B., L.V., and P.P. wrote the paper.

M.J.N. was supported by the Programme de bourses d'excellence Eiffel, and the Fonds de dotation Patrick de Brou de Laurière. The basic results here have been presented previously in abstract form (Nelson, Bosch, Venance and Pouget, 2010 Soc Neurosci Abstr).

Correspondence should be addressed to Matthew J. Nelson, CR-ICM, UMRS 975, INSERM - Université Pierre et Marie Curie, Neurologie et Thérapeutique Expérimentale, Hôpital de la Salpêtrière, 47 boulevard de I'Hôpital, 75651 Paris CEDEX 13, France. E-mail: matthew.nelson@icm-institute.org.

C. Bosch's present address: Department of Anatomy, Brain Health Research Center, University of Otago, PO Box 913, Dunedin 9054, New Zealand.

DOI:10.1523/JNEUROSCI.3502-12.2013

Copyright $\odot 2013$ the authors $\quad 0270-6474 / 13 / 332821-07 \$ 15.00 / 0$ would be considered to represent a "democratic" summation of the electrical potential contributions resulting geometrically from sources and sinks everywhere in the brain. However, if the microscale inhomogeneities inherent to neural tissue had an appreciable effect on the propagation of electrical signals, this would not be the case. If, for example, the presence or absence of a nearby fiber bundle were shown to affect the contribution of a given source or sink to the potential at other locations in the extracellular space, it would reject this assumption and change this view of the nature of LFPs.

LFP data have led to important advances in our understanding of brain function (O'Keefe and Recce, 1993; Schroeder et al., 1998; Fries et al., 2001; Liu and Newsome, 2006; Pesaran et al., 2002, 2008; Krieman et al., 2006; Womelsdorf et al., 2006; Chalk et al., 2010; Rutishauser et al., 2010; Liebe et al., 2012) and will continue to do so in the future. Despite this, many of the details surrounding the proper interpretation of LFPs are only limitedly understood and are current areas of active research (Logothetis et al., 2007; Katzner et al., 2009; Xing et al., 2009; Kajikawa and Schroeder, 2011; Lindén et al., 2011). Indeed, being able to better interpret these signals is an important step toward improving our understanding of brain function. Recent studies have focused on estimating the precise spatial extent of the spread of LFP signals from their sources (Katzner et al., 2009; Xing et al., 2009; Kajikawa and Schroeder, 2011; Lindén et al., 2011), but have entirely missed the major point that we address here-how do microscale inhomogeneities in the brain affect the propagation of LFPs? In an older work, David A. Robinson (1968) described how such inhomogeneities, specifically the ubiquitous presence of glial cells in brain tissue, would be expected to considerably impact the extracellular recording of spiking activity. Robinson 

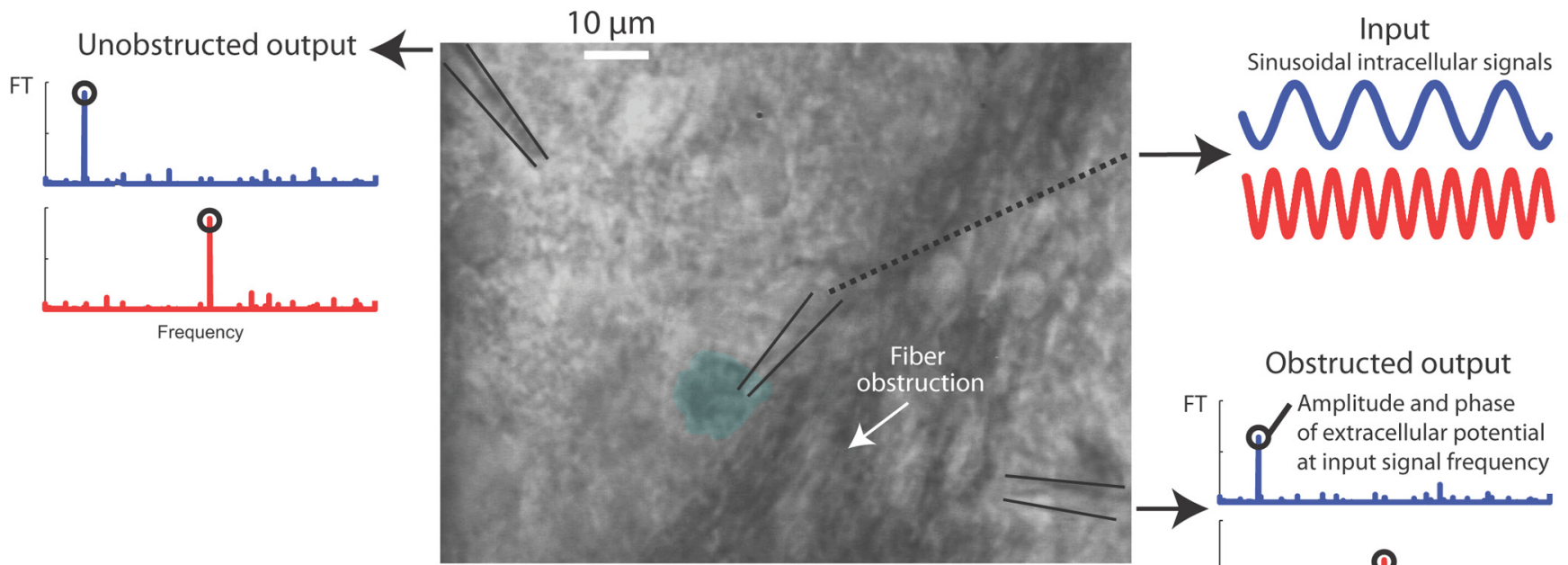

$10 \mu \mathrm{m}$
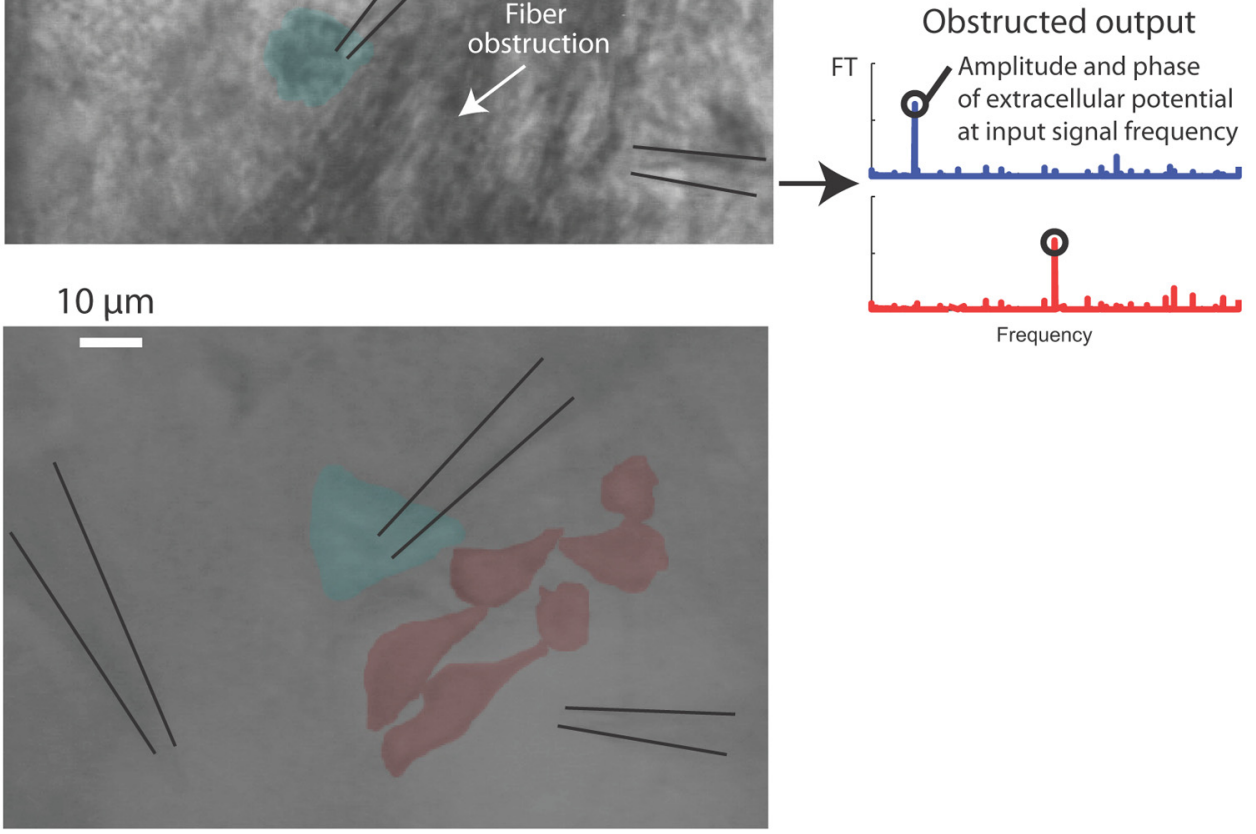

Figure 1. Diagram of experiment. The top and bottom show infrared microscope images taken during sample experiments with a fiber bundle obstruction and a group of cell bodies' obstruction, respectively. Black bars outline the location of the pipettes. The patched cell in each panel has been shaded green. In the bottom panel, the extracellular obstruction cell bodies have been shaded red. Sine wave stimuli were introduced intracellularly, then simultaneously measured extracellularly at the two locations in the slice. The amplitude of the extracellular voltage signal at the frequency of the input signal was well separated from the surrounding noise.

(1968), however, provides only anecdotal evidence to support his claim, and the effect of microscale inhomogeneities has until now never been directly tested experimentally.

\section{Materials and Methods}

Recording procedures. All experiments were performed in accordance with local animal welfare committee (Center for Interdisciplinary Research in Biology) and European Union guidelines (directive 86/609/ EEC). Patch-clamp recordings of striatal neurons were performed in horizontal brain slices $(330 \mu \mathrm{m})$ from Oncins France Strain A (OFA) rats (Charles River; postnatal days $\mathrm{P}_{17}-\mathrm{P}_{25}$ ) of either sex, using procedures described previously (Fino et al., 2005). Using a temperature control system (Bathcontroller V, Luigs \& Neumann) recordings were performed at $34^{\circ} \mathrm{C}$ or $26^{\circ} \mathrm{C}$. Slices were bathed in and continuously superfused at $2-3 \mathrm{ml} / \mathrm{min}$ with an extracellular solution similar to artificial CSF. The composition was as follows (in mM): $125 \mathrm{NaCl}, 2.5 \mathrm{KCl}, 25$ glucose, $25 \mathrm{NaHCO}_{3}, 1.25 \mathrm{NaH}_{2} \mathrm{PO}_{4}, 2 \mathrm{CaCl}_{2}, 1 \mathrm{MgCl}_{2}, 10 \mu \mathrm{M}$ pyruvic acid bubbled with $95 \% \mathrm{O}_{2}$ and $5 \% \mathrm{CO}_{2}$. Three borosilicate glass pipettes of 9-15 $\mathrm{M} \Omega$ impedance were used during the experiment, one to patch an individual cell and two to perform simultaneous extracellular voltage recordings at nearby locations in the slice. The intracellular pipette was filled with the following (in mM): $105 \mathrm{~K}$-gluconate, $30 \mathrm{KCl}, 10 \mathrm{HEPES}, 10$ phosphocreatine, 4 ATP-Mg, 0.3 GTP-Na, 0.3 EGTA (adjusted to $\mathrm{pH}$ 7.35 with $\mathrm{KOH}$ ). The extracellular pipettes were filled with the same solution used to bathe the slice. Recordings were made with EPC 10-3 amplifiers (HEKA Elektronik) with a very-high-input impedance $(\sim 1$ $\mathrm{T} \Omega$ ) to ensure there was no appreciable signal distortion imposed by the high-impedance electrodes (Nelson et al., 2008). For all experiments, a circular reference electrode surrounding the slice was used to avoid biasing current travel in any direction.

During the experiment, individual neurons and the microscale local composition of the extracellular space were identified using infrareddifferential interference contrast microscopy with a CCD camera (Optronis VX45). A target cell was first chosen based on the availability of two adequate extracellular recording locations relative to the cell. The extracellular locations were chosen to provide, at approximately the same distances from the patched cell, a sufficiently large contrast between the two locations in the severity of obstructions that were visible in the microscope images along their extracellular paths from the target cell. The two extracellular recording electrodes were positioned in these locations, and the target cell was then patch-clamped with the intracellular electrode. Sinusoidal stimuli were then introduced intracellularly to the patched cell while simultaneously recording the voltages extracellularly in the two locations. The median distance between the patched cell and the extracellular recording electrodes was $65 \mu \mathrm{m}$. Images of example recordings, and a diagram of the experiment performed are shown in Figure 1.

Immediately following the experiment, microscope images were digitally recorded every $5-10 \mu \mathrm{m}$ above and below the patched cell to verify offline the visible obstructions along the extracellular path between the patched cell and each extracellular pipette. Visible obstructions typically included cell bodies and clusters of cell bodies, axonal fiber bundles and blood vessels. We focused on axonal fiber bundle and cell body obstructions, performing 18 recordings with axonal fiber bundles as the most prominent obstruction, and 33 recordings with cell bodies as the most 
prominent obstruction. For the image correlation analyses (see Image analysis, below) we included data from one recording with a blood vessel as the primary obstruction. The same hardware channel was always used to perform the intracellular patch. The obstructed and unobstructed status of the two extracellular hardware channels were balanced across experiments individually within both the group of fiber bundle obstruction recordings and the group of cell body obstruction recordings. Neurons were identified as striatal output neurons $(44 \%, n=23)$ or different types of striatal interneurons and glial cells based on apparent cell morphology, current-voltage relationships, and specific firing patterns (Fino et al., 2005, 2008). The results of extracellular obstructions were not found to differ between the different types of patched cells.

Stimuli. Sine waves of 20 different frequencies were tested, varying approximately evenly on a logarithmic scale ranging from $6 \mathrm{~Hz}$ to 16.7 $\mathrm{kHz}$. Stimuli from 6 to $1282 \mathrm{~Hz}$ were sampled at $16.7 \mathrm{kHz}$; stimuli $>1282$ $\mathrm{Hz}$ were sampled at $50 \mathrm{kHz}$. Due to the occasional loss of the patch during the course of the experiment, not all frequencies were collected for every recording. A total of 100-300 traces of 100-1500 ms in length were averaged before recording the data to disk for offline analyses. Longer stimulus lengths and more traces were necessary for the low-frequency stimuli. For some recordings, the stimuli were presented in order of increasing frequency, but for the majority of the recordings the order of the presentation of the frequencies was randomized. Results were similar for each order of frequency presentation.

Stimuli were introduced with the intracellular electrode in currentclamp mode for most experiments, and in voltage-clamp mode for several recordings. Results between the two methods were similar and the data are thus pooled together. The injected current amplitudes for both clamping modes ranged from 200 to $300 \mathrm{pA}$. At stimulus frequencies of $2.5 \mathrm{kHz}$ and above, current amplitudes were progressively lower as a result of high-cut filters with a cutoff frequency of $10 \mathrm{kHz}$ applied to the stimulus. These lower stimulus amplitudes at high frequencies should be kept in mind as an additional variable when comparing between low and very high frequencies in our data, although the validity of any individual effect within any particular frequency group is not affected by this. Importantly, the extracellularly recorded voltages were completely unfiltered before their recording. Before conducting experiments, we verified via control recordings with an external signal generator in the bath without a slice that any amplitude changes or phase shifts in the recordings across frequencies were negligible.

Analyses. Offline analyses were conducted in Matlab (MathWorks). We determined the amplitude and phase of each recorded digitized signal by first averaging the waveform across all the cycle lengths of the known input frequency corresponding to that recording. The amplitude was then taken as $\sqrt{2}$ times the root mean square of the resulting waveform. The phase was determined by taking the four quadrant inverse tangent of a two-dimensional projection of the resulting waveform. The $x$ coordinate of the projection was the correlation (in the signal processing sense) of the resulting waveform with a cosine waveform, and the $y$ coordinate was the correlation with a cosine waveform shifted forward by 90 degrees. As indicated by the Fourier Transform of the sample recordings in Figure 1, the amplitude of the recorded extracellular signals at the frequency corresponding to the input stimulus was typically well separated from the noise. We tested several other methods to measure the amplitude and phase of the digitized signals, including a normalized Fourier Transform, all of which yielded near-identical results.

To view the effect of the obstructions, we normalized each recording as follows. Extracellular values were first normalized by the recorded estimates of the intracellular current. We then computed the average normalized voltages recorded for each extracellular hardware channel across all the experiments in each analysis, which included an equal number of recordings when the hardware channel was obstructed or unobstructed. Each individual extracellular recording was then normalized relative to this mean for its channel, and within-experiment comparisons were then performed across the extracellular channels using these normalized values. Normalization refers to dividing the original amplitude by the normalizing amplitude, and subtracting the normalizing phase from the original phase. For the within-experiment comparison for the amplitude results, the unobstructed channel's amplitude was subtracted from the obstructed channel's amplitude. Thus the amplitude results can be interpreted as the fraction of the average recording amplitude represented by each signal that was changed with the addition of the corresponding obstruction to the extracellular path. For the within-experiment comparison for the phase results, the unobstructed electrode's phase was subtracted from the obstructed electrode's phase. Thus the phase can be interpreted as the voltage phase shifts imposed by the obstruction.

We also investigated using a separate normalization procedure in which we compared the values from each channel to the corresponding values from a non-patch control recording in which the two extracellular electrodes were placed in a slice within a few micrometers of the normal intracellular electrode without a cell being patched. The results using this normalization were similar to the results we present here.

Statistics - mean and median effects. To test the mean and median differences, we pooled the data across low $(6-926 \mathrm{~Hz})$ and high $(1282 \mathrm{~Hz}$ to $16.7 \mathrm{kHz}$ ) frequencies and tested each group separately. Note that none of the results are critically dependent on the specific divisions between high and low frequencies. For amplitudes, we tested the significance of the mean differences with paired $t$ tests between obstructed and unobstructed electrodes across frequencies and experiments, and we tested the significance of the median differences with signed rank tests. For phases, we tested the significance of mean differences following procedures described by Fisher (1993), and we tested the significance of median differences using code taken from the circular statistics toolbox in Matlab (Berens, 2009).

Image analysis. The microscope images recorded during each session were analyzed offline to verify that the effects of the obstructions on the recorded signals correlated with the severity of the obstructions. The offline image scoring was done blindly, with the same experimenter scoring all of the sessions. Each extracellular electrode from each session received two scores from 0 to 9 reflecting the severity of the corresponding obstruction type along the extracellular path from the cell to the pipette tip. One score was given for the severity of axonal fiber bundle obstructions present, and one score was given for the severity of cell body obstructions present.

The within-experiment differences between the extracellular electrodes for the given obstruction type score was then correlated across sessions with the within-experiment normalized amplitude and phase differences between the electrodes (see Analyses, above). For the amplitudes, a nonparametric Spearman correlation was used. For the phases, a circular-linear correlation was performed, following procedures described by Fisher (1993, p. 161). There were 3 sessions for which the images could not be scored, and these were excluded from this analysis.

We also investigated other methods to score the images offline, including using a simple overall impression of the obstruction differences between the extracellular paths for each session, or by focusing on just the obstructions near the patched cell or near each extracellular pipette. All of these methods yielded similar results.

\section{Results}

We investigated the effect of microscale inhomogeneities of neural tissue on the propagation of electrical signals in the dorsal striatum. Indeed, the striatum is an optimal structure to analyze the effects of biological obstructions to extracellular electrical signal propagation since it is crossed by multiple fiber bundles and striatal output neurons displaying two patterns: either sparsely populated regions or packed groups of dozen of cells. This allowed for the comparison of unobstructed extracellular paths to paths obstructed with either axonal fibers or cell bodies. Moreover, in slices, $\sim 99 \%$ of striatal neurons are not tonically active, preventing additional sources of noise. We targeted striatal output neurons for whole-cell patch-clamp recording, as these neurons display highly branched dendrites that are restricted to an approximately spherical limited space with a 300-400 $\mu \mathrm{m}$ radius (Kawaguchi et al., 1989). This means that two extracellular electrodes placed at a similar distance from the soma, as per our experiment, will "see" a similar electrical impact of the patched 
cell's dendritic structure, which intracellularly carries the injected stimulus. We recorded from an experimental sample of 52 neurons overall, coming from 28 different animals. This sample size compares favorably to that used for recent publications using related methodologies (Trevelyan, 2009; Fröhlich and McCormick, 2010; Anastassiou et al., 2011). We performed a sufficient number of recordings for two different primary obstruction types to warrant specific analyses for them: bundles of axonal fibers $(n=18)$, and groups of cell bodies, including glial cells and other neurons $(n=33)$.

To demonstrate the overall effects of microscale obstructions on signal amplitudes, we plotted in Figure 2 the average across experiments of the obstructed electrode's recorded amplitude minus the unobstructed electrode's recorded amplitude within each experiment, normalized by the average recording's amplitude (see Materials and Methods). The resulting values reflect the fraction of the recorded signal that is changed based on the obstruction status of an extracellular measurement. Negative values on the plots reflect deleterious amplitude effects of the obstruction, e.g., when the obstructed electrodes had smaller amplitudes than the unobstructed electrodes, while positive values reflect facilitative effects, e.g., when the obstructed electrodes had larger amplitudes than the unobstructed electrodes. Along an extracellular path, the presence of fiber bundles led to deleterious amplitude effects over both lower frequencies [defined as 6-926 $\mathrm{Hz} ; n=181$ across experiments and frequencies; mean, -0.140 $(p<0.001)$; median, $-0.109(p<0.001)]$ and higher frequencies [defined as $1282 \mathrm{~Hz}$ to $16.7 \mathrm{kHz} ; n=124$ across experiments and frequencies; mean, -0.417 ( $p<0.001)$; median, -0.527 $(p<0.01)$ ] (Fig. $2 A)$. It seems likely that this effect may result from the myelination around the axonal fiber bundles, given the electrical properties of myelin (Weiss, 1997), which is indeed present in the types of slices we used for these experiments (Xiang et al., 2011). The presence of cell bodies led to deleterious amplitude effects over lower frequencies $[n=262$; mean, -0.156 ( $p<$ $0.001)$; median, $-0.054(p<0.01)]$, but facilitative effects at higher frequencies $[n=148$; mean, $0.328(p<0.05)$; median: $0.271(p<0.05)$ ] (Fig. $2 B)$. This frequency difference is consistent with existing expectations for the flow of current through biological tissue composed of cells dispersed in an interstitial fluid, including neural tissue (Grimnes and Martinsen, 2000).

If these obstructions truly impose a deleterious or facilitative effect on the propagation of signals as suggested in Figure 2, the magnitude of the amplitude effects imposed by a given obstruction should correlate with the severity of the obstruction. For example, when an electrical signal traverses a very large and dense bundle of axonal fibers, a larger amplitude drop should be observed than when the same signal traverses a smaller, sparser fiber bundle. Across experiments and frequencies, we correlated the differences between the obstructed and unobstructed electrode's recorded amplitudes with our estimation of the degree of the difference in severity of obstructions between them for each experiment. The latter was determined offline from inspection of infrared microscope images taken at a series of depths during the experiment. A separate estimate for each experiment was used for cell and fiber obstructions individually. Axonal fiber obstruction severity correlated with deleterious amplitude effects for both lower frequencies (Fig. 3A, top, Spearman's $\rho$ : $-0.097, p<0.05$, $n=419$ ) and higher frequencies (Fig. 3A, bottom, Spearman's $\rho$ : $-0.331, p<0.001, n=260$ ). Cell body obstruction severity was not correlated with any amplitude effects for lower frequencies (Fig. 3B, top, Spearman's $\rho: 0.081, p=0.096, n=419$ ), but was correlated with facilitative amplitude effects at higher frequencies (Fig. 3B, bottom, Spearman's $\rho: 0.134, p<0.05, n=260$ ). Together, these correlations are supportive of the overall amplitude effects in Figure 2, providing further evidence to support the result.

These amplitude changes imposed by obstructions might be accompanied by concomitant phase shifts. To assess the overall effects of microscale obstructions on signal phases, we plotted the average across experiments of the obstructed electrode's phase minus the unobstructed electrode's phase within each experiment, normalized by the average recording's phase relative to the stimulus (Fig. 4; see Materials and Methods). The resulting values reflect the change in phase of the extracellularly recorded voltage based on the obstruction status of an extracellular measurement. Positive (negative) values on the plots reflect the obstruction having a more capacitive (resistive) nature than the rest of the extracellular space. A phase near zero corresponds to no difference in the recorded phase between the obstructed and unobstructed electrodes. For both obstruction types and frequency ranges, we did not observe any phase effects in either direction [for fibers (Fig. 4A): Low frequencies, $n=181$; mean, 2.17 degrees $(~ p=$ 0.181 ); median, 1.421 degrees ( $p=0.747)$; High frequencies, $n=$ 124; mean, -2.10 degrees $(p=0.053)$; median, -1.68 degrees ( $p=0.530)$; for cells (Fig. $4 B)$ : Low frequencies, $n=262$; mean, -3.39 degrees $(p=0.070)$; median, 0.442 degrees $(p=0.951)$; High frequencies, $n=148$; mean, 4.34 degrees ( $p=0.275)$; median, 2.67 degrees $(p=0.217)$ ]. Collectively, these results show that microscale inhomogeneities affect the amplitude of contri- 

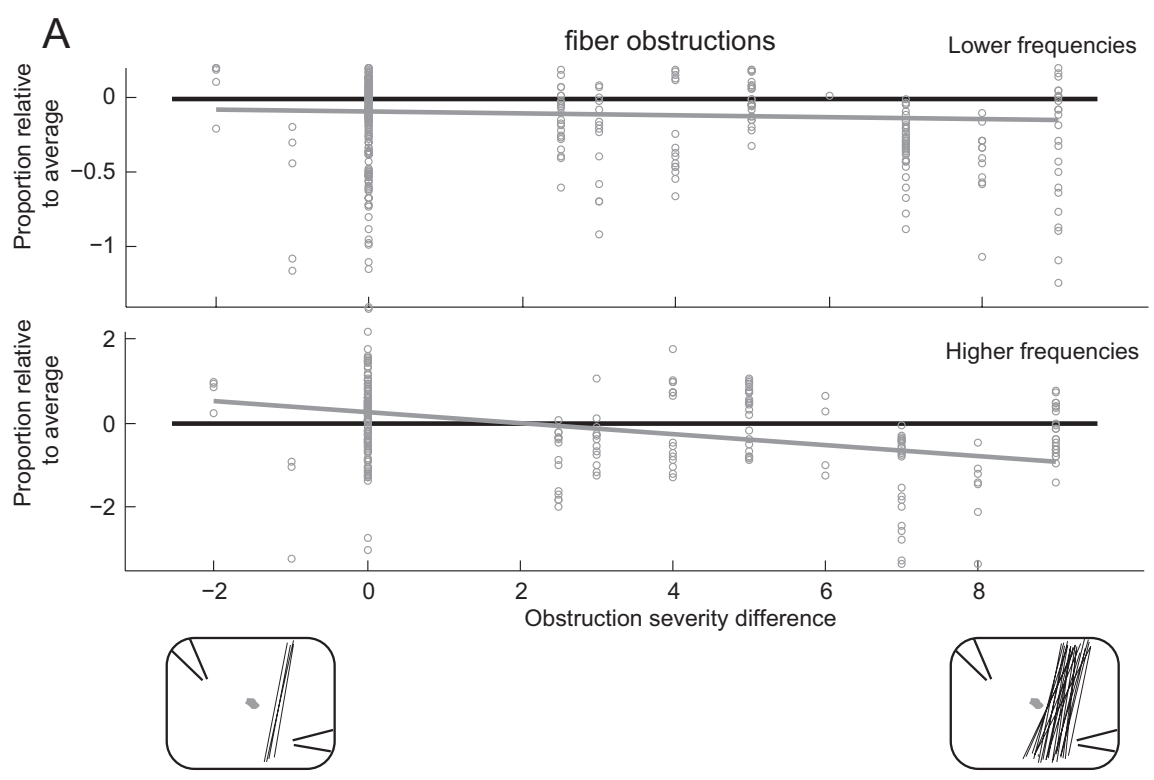

B
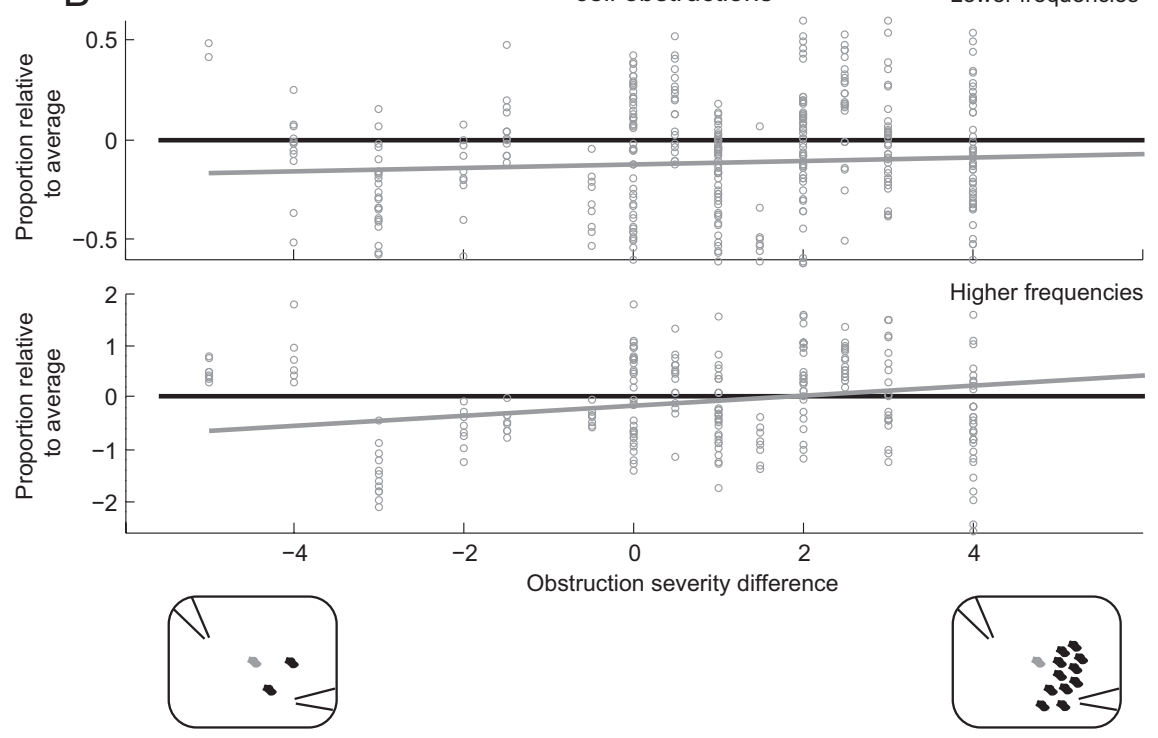

Figure 3. Correlations with obstruction severity. For each experiment and frequency, the normalized difference between the obstructed and unobstructed channel amplitudes is plotted against the difference between the two channels in the severity of the corresponding obstruction type, as assessed offline from inspection of the infrared microscope images of each experiment. $A$, Fiber bundle obstructions. $\boldsymbol{B}$, Cell body obstructions. The top panels of $\boldsymbol{A}$ and $\boldsymbol{B}$ correspond to lower frequency data, while the bottom panels correspond to higher frequency data. Linear regression lines are included. Some data exist outside the $y$-axis limits shown here. The visually assessed fiber obstruction severity correlates with the degree of deleterious amplitude effects over all frequencies. The cell body obstruction severity correlated with their facilitative amplitude effects at high frequencies.

butions to the extracellular potential from individual neural sources without altering their phases.

\section{Discussion}

We used simultaneous double extracellular measurements of sinusoidal stimuli induced in an individual neuron to determine whether microscale inhomogeneities that are inherent to neural tissue affect the propagation of electrical signals originating from a single cell. Axonal fiber bundles imposed deleterious amplitude effects at both low and high frequencies, both of which correlated with the severity of the fiber bundle obstruction. Cell bodies imposed deleterious amplitude effects at low frequencies and facilitative effects at high frequencies that correlated with the severity of the obstruction. However, no significant phase shifts were imposed by either obstruction.

The most important implications of our data are for the interpretation of measurements of extracellular potentials, specifically LFPs. We show that for the range of frequencies corresponding to LFP activity $(<200 \mathrm{~Hz})$, microscale obstructions impose deleterious amplitude effects to signals propagating across them. LFPs are thus not a democratic representation of the average activity of all the cells in a region. Instead, because of the cellular organization of the brain, certain neurons or neuron types would thus likely contribute more to the recorded LFPs than others based on differential presences of obstructions that tend to surround them. For example, gamma oscillations measured by both LFPs and electroencephalograms (EEGs) have been implicated to be involved in a wide variety of brain functions (Fries, 2009). Our results suggest these oscillations do not necessarily reflect an entrainment of all or even most cells in a given region at this frequency, but rather reflect particular neuron types as dictated by the microenvironment of a given area. This has implications for models of and our broader understanding of these oscillations. For similar reasons, these same effects may contribute to causing the spiking activity of certain neuron types to be recorded less often than others, as we also observed obstruction effects over the range of spiking frequencies.

Additionally, this work will be important to biophysicists and computational neuroscientists who model electrical signal propagation in the brain. Typically this has been modeled as electrically homogenous (Gold et al., 2006; Nunez and Srinivasan, 2006; Lindén et al., 2011), although the effect of the inhomogeneity of the extracellular space has been considered by some (Bédard et al., 2004; Bazhenov et al., 2011). This work provides the first direct experimental proof of effects related to these microscale inhomogeneities, as we show that electrically as well as physically, extracellular space in the brain indeed is not homogenous. Our results are in general agreement with those of Bédard et al. (2004) in that we find that inhomogeneity of the extracellular space leads to additional filtering and amplitude attenuation of the extracellular signal, though here we do not address the issue of the frequency dependence of extracellular signal propagation.

Readers should be aware that with our methodology, we could not visualize the detailed axonal and dendritic arborization of the patched neuron. The particular spatial distribution of the transmembrane currents of the patched neuron would be expected to affect the propagation of electrical signals, as has been implicated to occur for the extracellular propagation of action potential 
waveforms (Pettersen and Einevoll, 2008). However, we feel that this effect should balance out over the course of recordings, such that electrical signal propagation would not be expected to be systematically consistently biased toward either the obstructed or the unobstructed electrode. Thus, our results and conclusions hold without this detailed visualization of the patched neuron. We discuss this more in the Caveats and limitations subsection below.

The homogeneity question is one important line of inquiry of practical importance to the interpretation of LFPs. Other issues are also presently under debate and topics of current research. Such issues include the frequency dependence of signal propagation in the extracellular space (Bédard et al., 2004; Logothetis et al., 2007; Bédard and Destexhe, 2009), the overall relation between the extracellular voltage and intracellular events (Trevelyan, 2009; Bédard et al., 2010; Lindén et al., 2010), the degree of physical spread of LFPs from their neural sources (Katzner et al., 2009; Xing et al., 2009; Kajikawa and Schroeder, 2011; Lindén et al., 2011), and the role of electrode properties in measuring LFPs (Nunez and Srinivasan, 2006, Kay and Lazzara, 2010; Nelson and Pouget, 2010, 2012).

\section{Caveats and limitations}

In our experiment, there are a number of variables across sessions that we could not control for with this methodology that could conceivably influence the results of any given recording session in any given direction. Importantly though, such effects would cancel out over the balance of our experiments. For example, the morphology of the patched cells and their dendritic structures, which we cannot visualize online, may favor the transfer of current in particular directions. Another example of such an effect results from other obstructions to current flow that are not visible with this methodology but are certainly also present in the slice during these experiments, and could bias the propagation of signals in any particular direction over another. However, such effects would be expected to occur equally as often in favor of a particular result as they occur against it. The net effect should be that these simply provide additional sources of random interexperiment noise that effectively cancel out over the course of enough experiments. Indeed there are instances of individual recordings in which the extracellular pipette deemed to have the more obstructed path to the patched cell recorded larger rather than smaller amplitudes, perhaps due to a combination of the factors mentioned above. However, from the use of a sufficiently large sample of recordings, on average these results did indeed balance out over the course of all the experiments as we achieved statistically significant amplitude results. Our ability to effectively manage these sources of inter-experiment variability is considerably aided by the strength of our within-experiments experimental design, and the balancing across experiments of any conditions that we could control.

With respect to the question of cell morphology, importantly this potential effect would have been mitigated in our data by patching neurons with roughly spherical dendritic structures. Additionally, non-patch control recordings could also be done to circumvent the particular question of the effect of cell morphology. However, doing this would come at an inferential cost as the stimuli being tested would be considerably not natural, making it less clear as to whether or not the obstructions retain a considerable effect for natural stimuli. Here we prefer the inferences that can be made from using a relatively natural signal source, which can only be achieved using a patched cell as the source. Despite this, we do expect that we would find similar results using a non-patched signal source as well, although this variation of the experiment while monitoring microscale obstructions has not yet been done.

A possible concern is that the very extracellular obstructions that we target might tend to block the extracellular pipette with the more obstructed path to the target cell more often than the pipette with the more unobstructed path, leading to an artificial appearance of smaller signal amplitudes at the tip of the obstructed pipette. This was not likely to have had notice able effects on our data, however. The extracellular pipette impedances were measured and displayed continuously during the experiment and large increases in impedance after entering the slice were not observed. Moreover, the very high input impedance of the amplifiers that we used $(\sim 1 \mathrm{~T} \Omega)$, ensured that any nominal changes in the extracellular pipettes' impedance resulting from the accumulation of extracellular processes would not have affected the measurement of the voltage in the slice at the pipette's tip. Before beginning these experiments, we explicitly tested the dependence of our equipment's extracellular measurements on pipette impedances over a normal range of pipettes across frequencies using a procedure following what is described by Nelson et al. (2008). Over the range of frequencies we tested $(0.5 \mathrm{~Hz}$ to $12 \mathrm{kHz})$, pipette impedances are expected to change by a few orders of magnitude (Nelson et al., 2008), yet we still saw no change in the recorded signal across these frequencies. Thus the data we present here would have been independent of any reasonable fluctuations in pipette impedance.

We were limited to performing these experiments in brain slices to easily image the extracellular environment for placement 
of the extracellular pipettes into obstructed and unobstructed locations. However, we believe our demonstration of these effects in an ex vivo brain slice strongly indicates that the same basic effects would be present in the intact brain, even if there may be some quantitative differences in the magnitude of the effects. In fact it is possible that the effects would be even larger in an intact brain, given that in our slice preparation, current could travel out of the slice and through the bath, thus avoiding both obstructions and much of the tissue itself altogether. However, in an intact brain obstructions have the possibility to encapsulate electrical sources and sinks in all three dimensions. Further experiments in an intact brain, however, would be necessary to know the extent of these effects in that domain.

\section{References}

Anastassiou CA, Perin R, Markram H, Koch C (2011) Ephaptic coupling of cortical neurons. Nat Neurosci 14:217-223. CrossRef Medline

Bazhenov M, Lonjers P, Skorheim S, Bedard C, Destexhe A (2011) Nonhomogeneous extracellular resistivity affects the current-source density profiles of up-down state oscillations. Philos Trans R Soc A 369: 3802-3819. CrossRef

Bédard C, Destexhe A (2009) Macroscopic models of local field potentials and the apparent 1/f noise in brain activity. Biophys J 96:2589-2603. CrossRef Medline

Bédard C, Kröger H, Destexhe A (2004) Modeling extracellular field potentials and the frequency-filtering properties of extracellular space. Biophys J 86:1829-1842. CrossRef Medline

Bédard C, Rodrigues S, Roy N, Contreras D, Destexhe A (2010) Evidence for frequency-dependent extracellular impedance from the transfer function between extracellular and intracellular potentials: intracellular-LFP transfer function. J Comput Neurosci 29:389-403. CrossRef Medline

Berens P (2009) CircStat: a Matlab Toolbox for circular statistics. J Stat Softw 31:1-21.

Braitenberg V, Schuez A (1998) Cortex: statistics and geometry of neuronal connectivity, Ed 2. Berlin: Springer.

Chalk M, Herrero JL, Gieselmann MA, Delicato LS, Gotthardt S, Thiele A (2010) Attention reduces stimulus-driven gamma frequency oscillations and spike field coherence in V1. Neuron 66:114-125. CrossRef Medline

Fino E, Glowinski J, Venance L (2005) Bidirectional activity-dependent plasticity at corticostriatal synapses. J Neurosci 25:11279-11287. CrossRef Medline

Fino E, Deniau JM, Venance L (2008) Cell-specific spike-timing-dependent plasticity in GABAergic and cholinergic interneurons in corticostriatal rat brain slices. J Physiol 586:265-282. Medline

Fisher NI (1993) Statistical analysis of circular data. Cambridge, UK: Cambridge UP.

Fries P (2009) Neuronal gamma-band synchronization as a fundamental process in cortical computation. Annu Rev Neurosci 32:209-224. CrossRef Medline

Fries P, Reynolds JH, Rorie AE, Desimone R (2001) Modulation of oscillatory neuronal synchronization by selective visual attention. Science 291: 1560-1563. CrossRef Medline

Fröhlich F, McCormick DA (2010) Endogenous electric fields may guide neocortical network activity. Neuron 67:129-143. CrossRef Medline

Gold C, Henze DA, Koch C, Buzsáki G (2006) On the origin of the extracellular action potential waveform: a modeling study. J Neurophysiol 95: 3113-3128. CrossRef Medline

Grimnes S, Martinsen OG (2000) Bioimpedance and bioelectricity basics, pp 99-101. London: Academic.

Kajikawa Y, Schroeder CE (2011) How local is the local field potential? Neuron 72:847-858. CrossRef Medline

Katzner S, Nauhaus I, Benucci A, Bonin V, Ringach DL, Carandini M (2009) Local origin of field potentials in visual cortex. Neuron 61:35-41. CrossRef Medline

Kawaguchi Y, Wilson CJ, Emson PC (1989) Intracellular recording of identified neostriatal patch and matrix spiny cells in a slice preparation preserving cortical inputs. J Neurophysiol 62:1052-1068. Medline
Kay LM, Lazzara P (2010) How global are olfactory bulb oscillations? J Neurophysiol 104:1768-1773. CrossRef Medline

Krieman G, Hung CP, Kraskov A, Quiroga RQ, Poggio T, DiCarlo JJ (2006) Object selectivity of local field potentials and spikes in the macaque inferior temporal cortex. Neuron 49:35-41.

Liebe S, Hoerzer GM, Logothetis NK, Rainer G (2012) Theta coupling between $\mathrm{V} 4$ and prefrontal cortex predicts visual short-term memory performance. Nat Neurosci 15:456-462. CrossRef Medline

Lindén H, Pettersen KH, Einevoll GT (2010) Intrinsic dendritic filtering gives low-pass power spectra of local field potentials. J Comput Neurosci 29:423-444. CrossRef Medline

Lindén H, Tetzlaff T, Potjans TC, Pettersen KH, Grün S, Diesmann M, Einevoll GT (2011) Modeling the spatial reach of the LFP. Neuron 72 : 859-872. CrossRef Medline

Liu J, Newsome WT (2006) Local field potential in cortical area MT: stimulus tuning and behavioral correlations. J Neurosci 26:7779-7790. CrossRef Medline

Logothetis NK, Kayser C, Oeltermann A (2007) In vivo measurement of cortical impedance spectrum in monkeys: implications for signal propagation. Neuron 55:809-823. CrossRef Medline

Nelson MJ, Pouget P (2010) Do electrode properties create a problem in interpreting local field potential recordings? J Neurophysiol 103: 2315-2317. CrossRef Medline

Nelson MJ, Pouget P (2012) Physical model of coherent potentials measured with different electrode recording site sizes. J Neurophysiol 107: 1291-1300. CrossRef Medline

Nelson MJ, Pouget P, Nilsen EA, Patten CD, Schall JD (2008) Review of signal distortion through metal microelectrode recording circuits and filters. J Neurosci Methods 169:141-157. CrossRef Medline

Nunez PL, Srinivasan R (2006) Electric fields of the brain: The neurophysics of EEG, Ed 2. New York: Oxford UP.

O'Keefe J, Recce ML (1993) Phase-relationship between hippocampal place units and the EEG theta rhythm. Hippocampus 3:317-330. CrossRef Medline

Pesaran B, Pezaris JS, Sahani M, Mitra PP, Andersen RA (2002) Temporal structure in neuronal activity during working memory in macaque parietal cortex. Nat Neurosci 5:805-811. CrossRef Medline

Pesaran B, Nelson MJ, Andersen RA (2008) Free choice activates a decision circuit between frontal and parietal cortex. Nature 453:406-409. CrossRef Medline

Peters A, Palay SL, Webster HF (1991) The fine structure of the nervous system. Oxford: Oxford UP.

Pettersen KH, Einevoll GT (2008) Amplitude variability and extracellular low-pass filtering of neuronal spikes. Biophys J 94:784-802. CrossRef Medline

Robinson DA (1968) The electrical properties of metal microelectrodes. Proc IEEE 56:1065-1071. CrossRef

Rutishauser U, Ross IB, Mamelak AN, Schuman EM (2010) Human memory strength is predicted by theta-frequency phase-locking of single neurons. Nature 464:903-907. CrossRef Medline

Schroeder CE, Mehta AD, Givre SJ (1998) A spatiotemporal profile of visual system activation revealed by current source density analysis in the awake macaque. Cereb Cortex 8:575-592. CrossRef Medline

Trevelyan AJ (2009) The direct relationship between inhibitory currents and local field potentials. J Neurosci 29:15299-15307. CrossRef Medline

Weiss TF (1997) Cellular biophysics, pp 293-350. Cambridge, MA: MIT.

Womelsdorf T, Fries P, Mitra PP, Desimone R (2006) Gamma-band synchronization in visual cortex predicts speed of change detection. Nature 439:733-736. CrossRef Medline

Xiang Z, Valenza M, Cui L, Leoni V, Jeong HK, Brilli E, Zhang J, Peng Q, Duan W, Reeves SA, Cattaneo E, Krainc D (2011) Peroxisomeproliferator-activated receptor gamma coactivator 1 contributes to dysmyelination in experimental models of Huntington's Disease. J Neurosci 31:9544-9553. CrossRef Medline

Xing D, Yeh CI, Shapley RM (2009) Spatial spread of the local field potential and its laminar variation in visual cortex. J Neurosci 29:11540-11549. CrossRef Medline 